\title{
MASS EJECTION BY RADIATION PRESSURE
}

\author{
(Abstract)
}

\author{
ANNA ŻYTKOW \\ Institute of Astronomy, Polish Academy of Sciences, Warsaw, Poland
}

It was frequently suggested in recent years that the driving force for mass loss for the red supergiants may be the radiation pressure in continuum in the optically thick regions. There were many attempts to find selfconsistent models of time-independent mass outflow driven by this mechanism (Finzi and Wolf, 1971; Bisnovaty-Kogan and Nadyozhin, 1972; and many others); however, neither was satisfactory.

The aim of this paper is to indicate why radiation pressure in the high opacity regions should no longer be regarded as a promising mechanism in solving the problem of stationary mass ejection from the later type stars. The description of all the details may be found elsewhere (Żytkow, 1972, 1973).

In order to be able to treat the outflow as time-independent an element of matter undergoing acceleration should reach the velocity exceeding the escape velocity on a time-scale very short compared to the time required to eject the whole hydrogen rich envelope that can be expelled. In other words the reservoir of matter to be expelled should be much larger than the amount of matter actually undergoing acceleration. Let us mention that in the case of radiation pressure in continuum the basic assumption of time-independence was always made a priori with a merely superficial discussion of its validity. In view of our results it is this assumption which requires most careful consideration.

Within the framework of stationary outflow and spherical symmetry the models of outflowing envelopes were constructed by means of numerical integrations of equations of conservation of energy, momentum and mass together with radiative energy transport equation, equation of state including the effects of $\mathrm{H}$ and $\mathrm{He}$ ionization and $\mathrm{H}_{2}$ dissociation and proper opacity values. The solutions have to be regular in the singular point where the transition from a subsonic to a supersonic velocity takes place. In the photosphere the commonly used photospheric thermal condition $L=4 \pi \sigma r_{\text {phot }}^{2} T_{\text {phot }}^{4}$ (hereinafter all the symbols have their usual meaning) has to be satisfied. Thus, for a star mass $M$ the models of outflowing envelopes are fully determined by two free parameters, say: the luminosity $L$ of the star and the mass flux $F$. Obviously in this two parameter family of solutions only these leading to the expansion velocities that satisfy at infinity the condition $v_{\infty}^{2} \geqslant 0$ (for large radii $v_{\infty}^{2}=$ const $\left.=G M / r-v^{2} / 2\right)$ are relevant. Deep below the singular point in the (almost) static region of the models the properties of solutions should permit fitting of these models to the static interior (double-shell source carbon core in the case of low mass stars, helium core in the case of massive stars).

The models were constructed for $1 M_{\odot}$ and $30 M_{\odot}$ stars. The transition from 
subsonic to supersonic flow always takes place deep below the photosphere in the layers whose optical depth $\tau$ is large. So the upper limit to the rate at which momentum may be carried outwards to infinity is approximately $L \tau / c$, as may be derived from the equations of mass and momentum conservation. Thus the obtained rates of mass loss are rather large, ranging (approximately) from $10^{20}$ to $10^{25} \mathrm{~g} \mathrm{~s}^{-1}$. For a $1 M_{\odot}$ star the solutions satisfying the conditions stated above could be found for $L \gtrsim 1.625 \times 10^{4} L_{\odot}$, for a $30 M_{\odot}$ star for $L \gtrsim 1.15 \times 10^{6} L_{\odot}$. The models occupy well defined areas in the yellow supergiant regions on the HR diagram; within the scope of adopted assumptions for both masses the complete range of relevant $L$ and $F$ values was investigated.

Some properties of the models seem to suggest that these models may provide a realistic description of the phenomenon of mass loss. This line of approach should, however, be no longer maintained. The calculations show that although one formally can construct the models of stationary outflowing envelopes these models cannot be physically meaningful. The main problem is that the reservoir of (almost static) matter to be expelled is always comparable or even less than the amount of matter actually undergoing acceleration. So in the case of radiation pressure in continuum in the optically thick region one cannot get selfconsistent results. We would like to emphasize that it is not the whole star that constitutes the reservoir of matter to be expelled - we have at our disposal only these layers of the star that are above the hydrogen burning shell source, as in a realistic model of a mass losing star on one hand the matter would have to flow downwards into the core through the shell (in view of its burning in the shell source) and on the other hand the matter would have to flow outwards.

Our results suggest that if one wants to describe the mass loss phenomenon in terms of stationary outflow, one should look for mechanisms in which matter is mainly accelerated above or close to the photosphere. In this respect the most promising seems to be the model of mass loss in which the radiation pressure on dust is taken into account as suggested by Weymann (1962), Wickramasinghe et al. (1966), and discussed by Gilman (1972) and others.

\section{References}

Bisnovaty-Kogan, G. S. and Nadyozhin, D. K.: 1972, Astrophys. Space Sci. 15, 353.

Finzi, A. and Wolf, R. A.: 1971, Astron. Astrophys. 11, 418.

Gilman, R. C.: 1972, Astrophys. J. 178, 423.

Weymann, R.: 1962, Astrophys. J. 136, 476.

Wickramasinghe, N. C., Donn, B. D., and Stecher, T. P. : 1966, Astrophys. J. 146, 590.

Żytkow, A.: 1972, Acta Astron. 22, 103.

Żytkow, A.: 1973, Acta Astron. 23, 121. 